EUROPEAN JOURNAL OF PURE AND APPLIED MATHEMATICS

Vol. 13, No. 1, 2020, 130-143

ISSN 1307-5543 - www.ejpam.com

Published by New York Business Global

\title{
Simple Properties and Existence Theorem for the Henstock-Kurzweil-Stieltjes Integral of Functions Taking Values on $C[a, b]$ Space-valued Functions
}

\author{
Andrew Felix Cunanan ${ }^{1, *}$, Julius Benitez ${ }^{2}$ \\ ${ }^{1}$ Department of Natural Sciences and Mathematics, College of Arts and Sciences, \\ Surigao Del Sur State University, Tandag City, Surigao del Sur, Philippines \\ ${ }^{2}$ Department of Mathematics and Statistics, College of Sciences and Mathematics, \\ Mindanao State University-Iligan Institute of Technology, Tibanga, Iligan City, Philippines
}

\begin{abstract}
Henstock-Kurzweil integral, a nonabsolute integral, is a natural extension of the Riemann integral that was studied independently by Ralph Henstock and Jaroslav Kurzweil. This paper will introduce the Henstock-Kurzweil-Stieltjes integral of $\mathcal{C}[a, b]$-valued functions defined on a closed interval $[f, g] \subseteq \mathcal{C}[a, b]$, where $\mathcal{C}[a, b]$ is the space of all continuous real-valued functions defined on $[a, b] \subseteq \mathbb{R}$. Some simple properties of this integral will be formulated including the Cauchy criterion and an existence theorem will be provided.
\end{abstract}

2020 Mathematics Subject Classifications: 58C06,51M20, 26A42, 26B05, 26B30

Key Words and Phrases: $\mathcal{C}[a, b]$ space-valued function, $\delta$-fine tagged division, Henstock-KurzweilStieltjes integral, Continuity, Bounded variation.

\section{Introduction}

The Henstock-Kurzweil-Stieltjes integral is a generalized Riemann-Stieltjes integral which has properties similar to it. In the paper [9], Ubaidillah introduce the HenstockKurzweil integral of functions taking values in $\mathcal{C}[a, b]$ through Riemann sums

$$
S(F, D)=\sum_{D} F\left(t_{i}\right)\left[h_{i-1}, h_{i}\right]
$$

where $D=\left\{\left(\left[h_{i-1}, h_{i}\right], t_{i}\right)\right\}_{i=1}^{n}$ is a tagged division of $[f, g]$ Notion of integrals for Banach space-valued functions like Henstock integral for Banach space-valued functions, Henstock-Stieltjes integral of real-valued functions with respect to an increasing function and Henstock-Stieltjes integral for Banach spaces were already defined by Cao [3],

*Corresponding author.

DOI: https://doi.org/10.29020/nybg.ejpam.v13i1.3626

Email addresses: deofscunananiv@yahoo.com (A. Cunanan), julius.benitez@g.msuiit, edu.ph (J. Benitez) 
Lim [7] and Tikare [8], respectively. In this paper we change the way to define the domain of the function and the integrator. We shall choose first a closed interval $[f, g]$ as our domain and a continuous real-valued function $H$ instead of the identity map as our integrator.

\section{Preliminaries}

Throughout, we consider the space $\mathcal{C}[a, b]$ of all continuous real-valued functions defined on $[a, b]$. For more details of the space $\mathcal{C}[a, b]$, see [2], [5] or [9].

Let $[f, g]$ be a closed interval of $\mathcal{C}[a, b]$. A division of $[f, g]$ is any finite set $\left\{h_{0}, h_{1}, \ldots\right.$ ,$\left.h_{n}\right\} \subset[f, g]$ such that

$$
h_{0}=f, h_{n}=g \text { and } h_{i-1}<h_{i}
$$

for all $i=1,2, \ldots, n$. A tagged division of $[f, g]$ is a finite collection $\left\{\left(\left[h_{i-1}, h_{i}\right], t_{i}\right)\right.$ : $i=1,2, \ldots, n\}$ of interval-point pairs such that $\left\{h_{0}, h_{1}, \ldots, h_{n}\right\}$ is a division of $[f, g]$ and $t_{i} \in\left[h_{i-1}, h_{i}\right]$ for every $i=1,2, \ldots, n$. Each point $t_{i}$ is referred to as the tag of the corresponding subinterval $\left[h_{i-1}, h_{i}\right]$. Let $\theta$ be the null element in $\mathcal{C}[a, b]$, that is, $\theta(x)=0$, for all $x \in[a, b]$. A function $\delta:[f, g] \rightarrow \mathcal{C}[a, b]$ is said to be a gauge on $[f, g]$ if $\theta<\delta(h)$ for every $h \in[f, g]$.

Definition 1. [9] Let $\delta$ be a gauge on $[f, g]$. A tagged division $D=\left\{\left(\left[h_{i-1}, h_{i}\right], t_{i}\right): i=\right.$ $1,2, \ldots, n\}$ is said to be $\delta$-fine if

$$
t_{i} \in\left[h_{i-1}, h_{i}\right] \subset\left(t_{i}-\delta\left(t_{i}\right), t_{i}+\delta\left(t_{i}\right)\right)
$$

for every $i=1,2, \ldots, n$.

Theorem 1. [9] (Cousin's Lemma) If $\delta$ is a gauge on $[f, g] \subset \mathcal{C}[a, b]$, then there is a $\delta$-fine tagged division of $[f, g]$.

\section{Henstock-Kurzweil-Stieltjes Integral on $\mathcal{C}[a, b]$}

Let $D=\left\{\left(\left[h_{i-1}, h_{i}\right], t_{i}\right): i=1,2, \ldots, n\right\}$ be a tagged division of $[f, g]$ and $F, H$ : $[f, g] \rightarrow \mathcal{C}[a, b]$ be functions. We write

$$
S(F, H ; D)=\sum_{i=1}^{n} F\left(t_{i}\right)\left[H\left(h_{i}\right)-H\left(h_{i-1}\right)\right],
$$

called as Henstock-Kurzweil-Stieltjes sum of $F$ with respect to $H$ on $[f, g]$. For brevity, we write $D=\{([u, v], t)\}$ for a tagged division of $[f, g]$ and

$$
S(F, H ; D)=\sum_{D} F(t)[H(v)-H(u)] .
$$


Definition 2. Let $F, H:[f, g] \rightarrow \mathcal{C}[a, b]$ be functions. We say that the function $F$ is Henstock-Kurzweil-Stieltjes integrable with respect to $H$ on $[f, g]$ to $S \in \mathcal{C}[a, b]$, briefly $\mathcal{H K S}$-integrable, if for any $\epsilon>0$, there exists a gauge $\delta$ on $[f, g]$ such that for any $\delta$-fine tagged division $D$ of $[f, g]$, we have

$$
|S(F, H ; D)-S|<\epsilon \cdot e,
$$

where $e$ is the multiplicative identity in $\mathcal{C}[a, b]$. The element $S \in \mathcal{C}[a, b]$ is called Henstock-Kurweil-Stieltjes integral, briefly $\mathcal{H} \mathcal{K} \mathcal{S}$-integral, of $F$ with respect to $H$ on $[f, g]$ and is written by

$$
S=(\mathcal{H} \mathcal{K S}) \int_{f}^{g} F d H
$$

The collection of all functions which are $\mathcal{H} \mathcal{K} \mathcal{S}$-integrable with respect to $H$ on $[f, g]$ is denoted by $\mathcal{H} \mathcal{K} \mathcal{S}([f, g], H)$.

Theorem 2. (Uniqueness) If $F$ is $\mathcal{H} \mathcal{K S}$-integrable with respect to $H$ on $[f, g]$, then the $\mathcal{H K S}$-integral of $F$ with respect to $H$ on $[f, g]$ is unique.

Proof. Suppose that $F$ is $\mathcal{H} \mathcal{K} \mathcal{S}$-integrable with respect to $H$ on $[f, g]$ to $S_{1} \in \mathcal{C}[a, b]$ and $S_{2} \in \mathcal{C}[a, b]$. Let $\epsilon>0$. Then there exists a gauge $\delta_{1}$ on $[f, g]$ such that for all $\delta_{1}$-fine tagged division $D=\left\{\left(\left[h_{i-1}, h_{i}\right], t_{i}\right): i=1,2, \ldots, n\right\}$ of $[f, g]$, we have

$$
\left|S(F, H ; D)-S_{1}\right|<\frac{\epsilon}{2} \cdot e .
$$

Similarly, there exists a gauge $\delta_{2}$ on $[f, g]$ such that for all $\delta_{2}$-fine tagged division $Q=$ $\left\{\left(\left[k_{i-1}, k_{i}\right], s_{i}\right): i=1,2, \ldots, m\right\}$ of $[f, g]$, we have

$$
\left|S(F, H ; Q)-S_{2}\right|<\frac{\epsilon}{2} \cdot e .
$$

Define a function $\delta:[f, g] \rightarrow \mathcal{C}[a, b]$ by $\delta=\delta_{1} \wedge \delta_{2}$. Hence, by (1) and (2)

$$
\left|S_{1}-S_{2}\right|<\frac{\epsilon}{2} \cdot e+\frac{\epsilon}{2} \cdot e=\epsilon \cdot e .
$$

This shows that $S_{1}=S_{2}$. Therefore, the $\mathcal{H} \mathcal{K} \mathcal{S}$-integral of $F$ with respect to $H$ on $[f, g]$ is unique.

\section{Simple Properties}

Theorem 3. If $F, G \in \mathcal{H} \mathcal{K S}([f, g], H)$ and $\alpha \in \mathbb{R}$, then

(i) Homogenity: $\alpha \cdot F \in \mathcal{H K S}([f, g], H)$ and

$$
(\mathcal{H K S}) \int_{f}^{g}(\alpha \cdot F) d H=\alpha \cdot(\mathcal{H K S}) \int_{f}^{g} F d H .
$$


(ii) Linearity: $F+G \in \mathcal{H} \mathcal{K S}([f, g], H)$ and

$$
(\mathcal{H K S}) \int_{f}^{g}(F+G) d H=(\mathcal{H} \mathcal{K S}) \int_{f}^{g} F d H+(\mathcal{H K S}) \int_{f}^{g} G d H
$$

Proof.

(i) Let $\epsilon>0$. Then there exists a gauge $\delta$ on $[f, g]$ such that for any $\delta$-fine tagged division $D=\left\{\left(\left[h_{i-1}, h_{i}\right], t_{i}\right): i=1,2, \ldots, n\right\}$ of $[f, g]$, we have

$$
\left|\sum_{i=1}^{n} F\left(t_{i}\right)\left[H\left(h_{i}\right)-H\left(h_{i-1}\right)\right]-(\mathcal{H K S} \mathcal{S}) \int_{f}^{g} F d H\right|<\frac{\epsilon}{|\alpha|+1} \cdot e .
$$

Thus, for any $\delta$-fine tagged division $D=\left\{\left(\left[h_{i-1}, h_{i}\right], t_{i}\right): i=1,2, \ldots, n\right\}$ of $[f, g]$

$$
\begin{aligned}
\mid \sum_{i=1}^{n} & (\alpha \cdot F)\left(t_{i}\right)\left[H\left(h_{i}\right)-H\left(h_{i-1}\right)\right]-\alpha \cdot(\mathcal{H K \mathcal { S }}) \int_{f}^{g} F d H \mid \\
& =\left|\alpha\left\{\sum_{i=1}^{n} F\left(t_{i}\right)\left[H\left(h_{i}\right)-H\left(h_{i-1}\right)\right]-(\mathcal{H} \mathcal{K} \mathcal{S}) \int_{f}^{g} F d H\right\}\right| \\
& =|\alpha| \cdot\left|\sum_{i=1}^{n} F\left(t_{i}\right)\left[H\left(h_{i}\right)-H\left(h_{i-1}\right)\right]-(\mathcal{H} \mathcal{K S}) \int_{f}^{g} F d H\right| \\
& <|\alpha| \cdot \frac{\epsilon}{|\alpha|+1} \cdot e \\
& <\epsilon \cdot e .
\end{aligned}
$$

This shows that $\alpha \cdot F \in \mathcal{H} \mathcal{K S}([f, g], H)$ and

$$
(\mathcal{H K S}) \int_{f}^{g}(\alpha \cdot F) d H=\alpha \cdot(\mathcal{H K S}) \int_{f}^{g} F d H .
$$

(ii) Let $\epsilon>0$. Then there exists gauge $\delta_{F}$ on $[f, g]$ such that for any $\delta_{F}$-fine tagged division $D=\left\{\left(\left[h_{i-1}, h_{i}\right], t_{i}\right): i=1,2, \ldots, n\right\}$ of $[f, g]$, we have

$$
\left|\sum_{i=1}^{n} F\left(t_{i}\right)\left[H\left(h_{i}\right)-H\left(h_{i-1}\right)\right]-(\mathcal{H K S}) \int_{f}^{g} F d H\right|<\frac{\epsilon}{2} \cdot e .
$$

Similarly, there exists gauge $\delta_{G}$ on $[f, g]$ such that for any $\delta_{G}$-fine tagged division $Q=\left\{\left(\left[k_{i-1}, k_{i}\right], s_{i}\right): i=1,2, \ldots, m\right\}$ of $[f, g]$, we have

$$
\left|\sum_{i=1}^{m} G\left(s_{i}\right)\left[H\left(k_{i}\right)-H\left(k_{i-1}\right)\right]-(\mathcal{H K S}) \int_{f}^{g} G d H\right|<\frac{\epsilon}{2} \cdot e .
$$


Define $\delta=\delta_{F} \wedge \delta_{G}$. Then $\delta$ is a gauge on $[f, g]$. Let $D=\left\{\left(\left[h_{i-1}, h_{i}\right], t_{i}\right): i=\right.$ $1,2, \ldots, n\}$ be a $\delta$-fine tagged division of $[f, g]$. Then $D$ is both $\delta_{F}$ and $\delta_{G}$-fine. By (3) and (4),

$$
\begin{aligned}
& \left|\sum_{i=1}^{n}(F+G)\left(t_{i}\right)\left[H\left(h_{i}\right)-H\left(h_{i-1}\right)\right]-\left\{(\mathcal{H K S} \mathcal{S}) \int_{f}^{g} F d H+(\mathcal{H K S} \mathcal{S}) \int_{f}^{g} G d H\right\}\right| \\
& \leq\left|\sum_{i=1}^{n} F\left(t_{i}\right)\left[H\left(h_{i}\right)-H\left(h_{i-1}\right)\right]-(\mathcal{H K \mathcal { S }}) \int_{f}^{g} F d H\right| \\
& +\left|\sum_{i=1}^{n} G\left(t_{i}\right)\left[H\left(h_{i}\right)-H\left(h_{i-1}\right)\right]-(\mathcal{H K S}) \int_{f}^{g} G d H\right| \\
& <\frac{\epsilon}{2} \cdot e+\frac{\epsilon}{2} \cdot e=\epsilon \cdot e .
\end{aligned}
$$

Therefore, $F+G \in \mathcal{H} \mathcal{K S}([f, g], H)$ and

$$
(\mathcal{H K S}) \int_{f}^{g}(F+G) d H=(\mathcal{H} \mathcal{K S}) \int_{f}^{g} F d H+(\mathcal{H K S}) \int_{f}^{g} G d H
$$

Theorem 4. (Linearity of Integrator) If $F \in \mathcal{H K \mathcal { S }}\left([f, g], H_{1}\right) \cap \mathcal{H K \mathcal { K }}\left([f, g], H_{2}\right)$, then $F \in \mathcal{H} \mathcal{K S}\left([f, g], H_{1}+H_{2}\right)$ and

$$
(\mathcal{H K S}) \int_{f}^{g} F d\left(H_{1}+H_{2}\right)=(\mathcal{H} \mathcal{K S}) \int_{f}^{g} F d H_{1}+(\mathcal{H} \mathcal{K S}) \int_{f}^{g} F d H_{2} .
$$

Proof. Let $\epsilon>0$. Then there exists gauge $\delta_{H_{1}}$ on $[f, g]$ such that for any $\delta_{H_{1}}$-fine tagged division $D=\left\{\left(\left[h_{i-1}, h_{i}\right], t_{i}\right): i=1,2, \ldots, n\right\}$ of $[f, g]$, we have

$$
\left|\sum_{i=1}^{n} F\left(t_{i}\right)\left[H_{1}\left(h_{i}\right)-H_{1}\left(h_{i-1}\right)\right]-(\mathcal{H} \mathcal{K S}) \int_{f}^{g} F d H_{1}\right|<\frac{\epsilon}{2} \cdot e .
$$

Similarly, there exists gauge $\delta_{H_{2}}$ on $[f, g]$ such that for any $\delta_{H_{2}}$-fine tagged division $Q=$ $\left\{\left(\left[k_{i-1}, k_{i}\right], s_{i}\right): i=1,2, \ldots, m\right\}$ of $[f, g]$, we have

$$
\left|\sum_{i=1}^{m} F\left(s_{i}\right)\left[H_{2}\left(k_{i}\right)-H_{2}\left(k_{i-1}\right)\right]-(\mathcal{H} \mathcal{K S}) \int_{f}^{g} F d H_{2}\right|<\frac{\epsilon}{2} \cdot e .
$$

Define $\delta=\delta_{H_{1}} \wedge \delta_{H_{2}}$. Then $\delta$ is a gauge on $[f, g]$. Let $D=\left\{\left(\left[h_{i-1}, h_{i}\right], t_{i}\right): i=1,2, \ldots, n\right\}$ be a $\delta$-fine tagged division of $[f, g]$. Then $D$ is both $\delta_{H_{1}}$ and $\delta_{H_{2}}$-fine. By (5) and (6),

$$
\left|\sum_{i=1}^{n} F\left(t_{i}\right)\left[\left(H_{1}+H_{2}\right)\left(h_{i}\right)-\left(H_{1}+H_{2}\right)\left(h_{i-1}\right)\right]-\left\{(\mathcal{H K S}) \int_{f}^{g} F d H_{1}+(\mathcal{H} \mathcal{K S}) \int_{f}^{g} F d H_{2}\right\}\right|
$$




$$
\begin{aligned}
& \leq\left|\sum_{i=1}^{n} F\left(t_{i}\right)\left[H_{1}\left(h_{i}\right)-H_{1}\left(h_{i-1}\right)\right]-(\mathcal{H} \mathcal{K} \mathcal{S}) \int_{f}^{g} F d H_{1}\right| \\
& \quad+\left|\sum_{i=1}^{n} F\left(t_{i}\right)\left[H_{2}\left(h_{i}\right)-H_{2}\left(h_{i-1}\right)\right]-(\mathcal{H} \mathcal{K} \mathcal{S}) \int_{f}^{g} F d H_{2}\right| \\
& <\frac{\epsilon}{2} \cdot e+\frac{\epsilon}{2} \cdot e=\epsilon \cdot e .
\end{aligned}
$$

Therefore, $F \in \mathcal{H} \mathcal{K S}\left([f, g], H_{1}+H_{2}\right)$ and

$$
(\mathcal{H} \mathcal{K S}) \int_{f}^{g} F d\left(H_{1}+H_{2}\right)=(\mathcal{H} \mathcal{K S}) \int_{f}^{g} F d H_{1}+(\mathcal{H K S}) \int_{f}^{g} F d H_{2} .
$$

Theorem 5. (Additivity) Let $f \leq r \leq g$. If $F \in \mathcal{H} \mathcal{K S}([f, r], H)$ and $F \in \mathcal{H} \mathcal{K S}([r, g], H)$, then $F \in \mathcal{H} \mathcal{K S}([f, g], H)$ and

$$
(\mathcal{H} \mathcal{K S}) \int_{f}^{g} F d H=(\mathcal{H} \mathcal{K S}) \int_{f}^{r} F d H+(\mathcal{H} \mathcal{K S}) \int_{r}^{g} F d H
$$

Proof. Let $\epsilon>0$. Then there exists gauge $\delta_{1}$ on $[f, r]$ such that for any $\delta_{1}$-fine tagged division $D=\left\{\left(\left[h_{i-1}, h_{i}\right], t_{i}\right): i=1,2, \ldots, n\right\}$ of $[f, r]$, we have

$$
\left|\sum_{i=1}^{n} F\left(t_{i}\right)\left[H\left(h_{i}\right)-H\left(h_{i-1}\right)\right]-(\mathcal{H K S}) \int_{f}^{r} F d H\right|<\frac{\epsilon}{2} \cdot e .
$$

Similarly, there exists gauge $\delta_{2}$ on $[r, g]$ such that for any $\delta_{2}$-fine tagged division $Q=$ $\left\{\left(\left[k_{i-1}, k_{i}\right], s_{i}\right): i=1,2, \ldots, m\right\}$ of $[r, g]$, we have

$$
\left|\sum_{i=1}^{m} F\left(s_{i}\right)\left[H\left(k_{i}\right)-H\left(k_{i-1}\right)\right]-(\mathcal{H K S}) \int_{r}^{g} F d H\right|<\frac{\epsilon}{2} \cdot e .
$$

Define a function $\delta:[f, g] \rightarrow \mathcal{C}[f, g]$ by

$$
\delta(h)= \begin{cases}\delta_{1}(h) \wedge(r-h) & , \text { if } f \leq h \leq r \\ \delta_{1}(h \wedge r) \wedge \delta_{2}(h \vee r) & , \text { if } h=r \text { or } h \text { is incomparable to } r \\ \delta_{2}(h) \wedge(h-r) & , \text { if } r \leq h \leq g .\end{cases}
$$

Then $\delta$ is a gauge on $[f, g]$. Let $D=\left\{\left(\left[h_{i-1}, h_{i}\right], t_{i}\right): i=1,2, \ldots, n\right\}$ be a $\delta$-fine tagged division of $[f, g]$. By definition of $\delta$, we have $r=h_{i_{0}}$ for some $i_{0} \in\{1,2, \ldots, n\}$. Hence, $D=D_{1} \cup D_{2}$ for some $\delta_{1}$-fine tagged division $D_{1}$ of $[f, r]$ and $\delta_{2}$-fine tagged division $D_{2}$ of $[r, g]$. By (7) and (8),

$$
\left|\sum_{i=1}^{n} F\left(t_{i}\right)\left[H\left(h_{i}\right)-H\left(h_{i-1}\right)\right]-\left\{(\mathcal{H} \mathcal{K S}) \int_{f}^{r} F d H+(\mathcal{H} \mathcal{K S}) \int_{r}^{g} F d H\right\}\right|<\epsilon \cdot e .
$$


Therefore, $F \in \mathcal{H} \mathcal{K} \mathcal{S}([f, g], H)$ and

$$
(\mathcal{H} \mathcal{K S}) \int_{f}^{g} F d H=(\mathcal{H} \mathcal{K S}) \int_{f}^{r} F d H+(\mathcal{H K S}) \int_{r}^{g} F d H
$$

In the next theorem, we give an analogous form of Cauchy criterion for $\mathcal{H K \mathcal { K }}$-integral.

Theorem 6. (Cauchy Criterion) $F \in \mathcal{H K S}([f, g], H)$ if and only if for every $\epsilon>0$ there exists a gauge $\delta$ on $[f, g]$ such that for any $\delta$-fine tagged divisions $D=\{([u, v], t)\}$ and $Q=\left\{\left(\left[u^{\prime}, v^{\prime}\right], s\right)\right\}$ of $[f, g]$, we have

$$
\left|\sum_{D} F(t)[H(v)-H(u)]-\sum_{Q} F(s)\left[H\left(v^{\prime}\right)-H\left(u^{\prime}\right)\right]\right|<\epsilon \cdot e .
$$

Proof. $(\Rightarrow)$ Let $\epsilon>0$. Then there exists a gauge $\delta$ on $[f, g]$ such that for any $\delta$-fine tagged division $D=\{([u, v], t)\}$ of $[f, g]$, we have

$$
\left|\sum_{D} F(t)[H(v)-H(u)]-(\mathcal{H} \mathcal{K S}) \int_{f}^{g} F d H\right|<\frac{\epsilon}{2} \cdot e .
$$

Let $D=\{([u, v], t)\}$ and $Q=\left\{\left(\left[u^{\prime}, v^{\prime}\right], s\right)\right\}$ be any $\delta$-fine tagged divisions of $[f, g]$. By $(9)$

$$
\left|\sum_{D} F(t)[H(v)-H(u)]-\sum_{Q} F(s)\left[H\left(v^{\prime}\right)-H\left(u^{\prime}\right)\right]\right|<\epsilon \cdot e .
$$

$(\Leftarrow)$ By assumption, for each $n \in \mathbb{N}$, there exists a gauge $\delta_{n}$ on $[f, g]$ such that for any $\delta_{n}$-fine division $D=\{([u, v], t)\}$ and $Q=\left\{\left(\left[u^{\prime}, v^{\prime}\right], s\right)\right\}$ of $[f, g]$, we have

$$
\left|\sum_{D} F(t)[H(v)-H(u)]-\sum_{Q} F(s)\left[H\left(v^{\prime}\right)-H\left(u^{\prime}\right)\right]\right|<\frac{1}{n} \cdot e .
$$

We may assume that $\left\{\delta_{n}\right\}$ is decreasing; that is, $\delta_{n} \geq \delta_{n+1}$ for all $n$.

Now, for each $n \in \mathbb{N}$, fix a $\delta_{n}$-fine tagged division $D_{n}=\{([u, v], t)\}$ of $[f, g]$ and we write

$$
r_{n}=\sum_{D_{n}} F(t)[H(v)-H(u)] .
$$

Note that if $m \geq n$ then $\delta_{n} \geq \delta_{m}$; implying that every $\delta_{m}$-fine tagged division of $[f, g]$ is also a $\delta_{n}$-fine tagged division of $[f, g]$. Thus, for all $m>n$

$$
\left|r_{n}-r_{m}\right|=\left|\sum_{D_{n}} F(t)[H(v)-H(u)]-\sum_{D_{m}} F(s)\left[H\left(v^{\prime}\right)-H\left(u^{\prime}\right)\right]\right|<\frac{1}{n} \cdot e .
$$


Hence, $\left\{r_{n}\right\}$ is a Cauchy sequence in $\mathcal{C}[a, b]$. Since $\mathcal{C}[a, b]$ is complete, $\left\{r_{n}\right\}$ converges to some $r \in \mathcal{C}[a, b]$. We claim that

$$
r=(\mathcal{H K S}) \int_{f}^{g} F d H
$$

Let $\epsilon>0$. Since $\lim _{n \rightarrow \infty} r_{n}=r$ in $\mathcal{C}[a, b]$, there exists $N_{1} \in \mathbb{N}$ such that for any $n \geq N_{1}$,

$$
\left|r_{n}-r\right|<e \cdot \frac{\epsilon}{2}
$$

By Archimedean Principle, there exists $N_{2} \in \mathbb{N}$ such that $\frac{1}{N_{2}}<\frac{\epsilon}{2}$. Take $N=N_{1} \wedge N_{2}$. Define a gauge $\delta:[f, g] \rightarrow \mathcal{C}[a, b]$ by $\delta=\delta_{N}$. Let $D=\{([u, v], t)\}$ be any $\delta$-fine tagged division of $[f, g]$. Note that $D$ is also $\delta_{N}$-fine tagged division of $[f, g], N \geq N_{1}$ and $N \geq N_{2}$. Thus, by (10) and (11)

$$
\left|\sum_{D} F(t)[H(v)-H(u)]-r\right|<\epsilon \cdot e .
$$

This proves our claim.

Theorem 7. If $F \in \mathcal{H} \mathcal{K S}([f, g], H)$ and $[r, s] \subseteq[f, g]$, then $F \in \mathcal{H} \mathcal{K S}([r, s], H)$.

Proof. Let $\epsilon>0$. By Theorem 6, there exists a gauge $\delta$ on $[f, g]$ such that for any $\delta$-fine tagged divisions $D$ and $Q$ of $[f, g]$, we have

$$
\left|\sum_{D} F(t)[H(v)-H(u)]-\sum_{Q} F(t)[H(v)-H(u)]\right|<\epsilon \cdot e .
$$

Consider any $\delta$-fine tagged divisions $P_{1}$ and $P_{2}$ of $[r, s]$. If $D_{1}$ is any $\delta$-fine tagged division of $[f, r]$ and $D_{2}$ is any $\delta$-fine tagged division of $[s, g]$, then

$$
D=D_{1} \cup P_{1} \cup D_{2} \text { and } Q=D_{1} \cup P_{2} \cup D_{2}
$$

are $\delta$-fine tagged divisions of $[f, g]$ and by $(12)$

$$
\left|\sum_{P_{1}} F(t)[H(v)-H(u)]-\sum_{P_{2}} F(t)[H(v)-H(u)]\right|<\epsilon \cdot e .
$$

By Cauchy criterion, $F \in \mathcal{H} \mathcal{K} \mathcal{S}([r, s], H)$.

Theorem 8. Let $H:[f, g] \rightarrow \mathcal{C}[a, b]$ be increasing, that is, $H(k) \leq H(h)$ for any $k \leq h$ in $[f, g]$. If $F \in \mathcal{H} \mathcal{K S}([f, g], H)$ and $F(h) \geq \theta$ for every $h \in[f, g]$, then

$$
(\mathcal{H K S}) \int_{f}^{g} F d H \geq \theta .
$$


Proof. Let $\epsilon>0$. Then there exists a gauge $\delta$ on $[f, g]$ such that for any $\delta$-fine tagged division $D$ of $[f, g]$, we have

$$
\left|\sum_{D} F(t)[H(v)-H(u)]-(\mathcal{H} \mathcal{K S}) \int_{f}^{g} F d H\right|<\epsilon \cdot e .
$$

Since $F(h) \geq \theta$ for all $h \in[f, g]$ and $H$ is increasing,

$$
\sum_{D} F(t)[H(v)-H(u)] \geq \theta
$$

Therefore,

$$
\theta \leq \sum_{D} F(t)[H(v)-H(u)]<(\mathcal{H K S}) \int_{f}^{g} F d H+\epsilon \cdot e .
$$

Since $\epsilon>0$ is arbitrary,

$$
(\mathcal{H K S}) \int_{f}^{g} F d H \geq \theta
$$

Theorem 9. If $F, G \in \mathcal{H} \mathcal{K S}([f, g], H)$ and $F(h) \leq G(h)$, for all $h \in[f, g]$, then

$$
(\mathcal{H} \mathcal{K S}) \int_{f}^{g} F d H \leq(\mathcal{H} \mathcal{K S}) \int_{f}^{g} G d H
$$

Proof. Define a function $E$ on $[f, g]$ by setting $E(h)=G(h)-F(h)$, for all $h \in[f, g]$. Then $E(h) \geq \theta$, for all $h \in[f, g]$. Since $F, G \in \mathcal{H K S}([f, g], H), E \in \mathcal{H} \mathcal{K S}([f, g], H)$ and by Theorem 8

$$
(\mathcal{H K S}) \int_{f}^{g} E d H \geq \theta
$$

Hence,

$$
\theta \leq(\mathcal{H K S}) \int_{f}^{g} E d H=(\mathcal{H} \mathcal{K S}) \int_{f}^{g}(G-F) d H=(\mathcal{H} \mathcal{K S}) \int_{f}^{g} G d H-(\mathcal{H} \mathcal{K S}) \int_{f}^{g} F d H .
$$

Therefore,

$$
(\mathcal{H} \mathcal{K S}) \int_{f}^{g} G d H \leq(\mathcal{H K S}) \int_{f}^{g} F d H .
$$

\section{An Existence Theorem}

A function $F:[f, g] \rightarrow \mathcal{C}[a, b]$ is bounded on $[f, g]$ if there exists $K \geq \theta$ in $\mathcal{C}[a, b]$ such that

$$
|F(h)| \leq K, \text { for all } h \in[f, g] .
$$


A function $F:[f, g] \rightarrow \mathcal{C}[a, b]$ is continuous at $h_{0} \in[f, g]$, if for any $\epsilon>0$ there exists $\delta=\delta\left(h_{0}\right)>\theta$ such that whenever $h \in[f, g]$ with $\left|h-h_{0}\right|<\delta$, we have

$$
\left|F(h)-F\left(h_{0}\right)\right|<\epsilon \cdot e .
$$

$F$ is said to be uniformly continuous on $[f, g]$, if for any $\epsilon>0$ there exists $\delta>\theta$ such that whenever $h, h^{\prime} \in[f, g]$ with $\left|h^{\prime}-h\right|<\delta$, we have

$$
\left|F\left(h^{\prime}\right)-F(h)\right|<\epsilon \cdot e .
$$

If $F:[f, g] \rightarrow \mathcal{C}[a, b]$ is uniformly continuous on $[f, g]$, then it is continuous on $[f, g]$.

Definition 3. Let $D_{1}$ and $D_{2}$ be tagged divisions of $[f, g]$. We say that $D_{2}$ is finer than $D_{1}$, denoted by $D_{1} \ll D_{2}$, if for every $([u, v], t) \in D_{2}$ there exists $\left(\left[u^{\prime}, v^{\prime}\right], t^{\prime}\right) \in D_{1}$ such that $[u, v] \subseteq\left[u^{\prime}, v^{\prime}\right]$, and every tag in $D_{1}$ is a tag in $D_{2}$. For every $\left(\left[u^{\prime}, v^{\prime}\right], t^{\prime}\right) \in D_{1}$, the tagged division $P=\left\{\left(\left[z_{i-1}, z_{i}\right], t_{i}\right) \in D_{2}:\left[z_{i-1}, z_{i}\right] \subseteq\left[u^{\prime}, v^{\prime}\right], i=1,2, \ldots, n\right\}$ is the refinement of $\left(\left[u^{\prime}, v^{\prime}\right], t^{\prime}\right)$ in $D_{2}$.

We can easily see that if $D_{1}$ and $D_{2}$ are tagged divisions of $[f, g]$, then there exists a tagged division $D_{0}$ of $[f, g]$ such that $D_{1} \ll D_{0}$ and $D_{2} \ll D_{0}$.

Let $\mathcal{D}([f, g])$ be the collection of all divisions of $[f, g]$. For $F:[f, g] \rightarrow \mathcal{C}[a, b]$ and $D=\{[u, v]\} \in \mathcal{D}([f, g])$, the variation of $F$ over $D$ is given by

$$
\boldsymbol{v a r}(F, D)=\sum_{D}|F(v)-F(u)|
$$

Note that for any division $D$ of $[f, g], \boldsymbol{v a r}(F, D)$ is a continuous function on $[a, b]$; that is, $\boldsymbol{v a r}(F, D) \in \mathcal{C}[a, b]$, for any $D \in \mathcal{D}([f, g])$.

Definition 4. We say that the function $F:[f, g] \rightarrow \mathcal{C}[a, b]$ is of bounded variation on $[f, g]$ if

$$
\boldsymbol{v}_{F}=\boldsymbol{v}(F ;[f, g])=\sup _{D \in \mathcal{D}([f, g])} \boldsymbol{v a r}(F, D)
$$

is continuous on $[a, b]$; that is, $\boldsymbol{v}_{F} \in \mathcal{C}[a, b]$.

Note that for any $F:[f, g] \rightarrow \mathcal{C}[a, b], v_{F}$ is a mapping from $[a, b]$ to $[0,+\infty]$; that is,

$$
0 \leq \boldsymbol{v}_{F}(x) \leq+\infty, \text { for all } x \in[a, b] .
$$

Hence, if $F:[f, g] \rightarrow \mathcal{C}[a, b]$ is of bounded variation, then

$$
0 \leq \boldsymbol{v}_{F}(x)<+\infty, \text { for all } x \in[a, b] .
$$

Theorem 10. Let $H:[f, g] \mapsto \mathcal{C}[a, b]$ be of bounded variation. Then the variation of $H$ is additive; that is, if $f \leq r \leq g$, then

$$
\boldsymbol{v}(H ;[f, g])=\boldsymbol{v}(H ;[f, r])+\boldsymbol{v}(H ;[r, g]) .
$$


Proof. Suppose that $H:[f, g] \rightarrow \mathcal{C}[a, b]$ is of bounded variation. Let $r \in[f, g]$ and $D=\left\{h_{0}, \ldots, h_{n}\right\}$ be a division of $[f, g]$. Then $D^{\prime}=\left\{h_{0}, \ldots, h_{k-1}, r, h_{k}, \ldots, h_{n}\right\}$ is a refinement of $D$ obtained by adjoining $r$ to $D$. Thus

$$
\sum_{D}|H(v)-H(u)| \leq \sum_{D_{1}}|H(v)-H(u)|+\sum_{D_{2}}|H(v)-H(u)|
$$

where $D_{1}=\left\{f=h_{0}, h_{1}, \ldots, h_{k-1}, r\right\}$ and $D_{2}=\left\{r, h_{k}, \ldots, h_{n}=g\right\}$. Note that $D^{\prime}=$ $D_{1} \cup D_{2}$ and that

$$
\begin{aligned}
& \sum_{D_{1}}|H(v)-H(u)| \leq \sup _{D \in \mathcal{D}([f, r])}\left(\sum_{D}|H(v)-H(u)|\right)=\boldsymbol{v}(H ;[f, r]) \text { and } \\
& \sum_{D_{2}}|H(v)-H(u)| \leq \sup _{D \in \mathcal{D}([r, g])}\left(\sum_{D}|H(v)-H(u)|\right)=\boldsymbol{v}(H ;[r, g]) .
\end{aligned}
$$

Hence,

$$
\boldsymbol{v}(H ;[f, g])=\sup _{D \in \mathcal{D}([f, g])}\left(\sum_{D}|H(v)-H(u)|\right) \leq \boldsymbol{v}(H ;[f, r])+\boldsymbol{v}(H ;[r, g]) .
$$

On the other hand, for any $D_{1} \in \mathcal{D}([f, r])$ and $D_{2} \in \mathcal{D}([r, g])$, their union $D^{\prime}=$ $D_{1} \cup D_{2} \in \mathcal{D}_{r}([f, g])$, where $\mathcal{D}_{r}([f, g])$ is the set of all divisions of $[f, g]$ with $r$ as one of the division points. Note that $\mathcal{D}_{r}([f, g]) \subseteq \mathcal{D}([f, g])$. Hence,

$$
\sup _{D^{\prime} \in \mathcal{D}_{r}([f, g])}\left(\sum_{D^{\prime}}|H(v)-H(u)|\right) \leq \sup _{D \in \mathcal{D}([f, g])}\left(\sum_{D}|H(v)-H(u)|\right)=\boldsymbol{v}(H ;[f, g])
$$

Thus,

$$
\begin{aligned}
\boldsymbol{v}(H ;[f, r])+\boldsymbol{v}(H ;[r, g]) & \leq \sup _{D^{\prime} \in \mathcal{D}_{r}([f, g])}\left(\sum_{D^{\prime}}|H(v)-H(u)|\right) \\
& \leq \boldsymbol{v}(H ;[f, g]) .
\end{aligned}
$$

Therefore, combining the two inequalities

$$
\boldsymbol{v}(H ;[f, r])+\boldsymbol{v}(H ;[r, g])=\boldsymbol{v}(H ;[f, g]) .
$$

Theorem 11. (Existence Theorem) If $F:[f, g] \rightarrow \mathcal{C}[a, b]$ is continuous and $H:[f, g] \rightarrow$ $\mathcal{C}[a, b]$ is of bounded variation on $[f, g]$, then $F \in \mathcal{H} \mathcal{K} \mathcal{S}([f, g], H)$.

Proof. Let $\epsilon>0$. Since $H$ is of bounded variation, $\boldsymbol{v}_{H} \in \mathcal{C}[a, b]$. This means that there exists $K>0$ such that $\boldsymbol{v}_{H}(x) \leq K$ for all $x \in[a, b]$. Since $F$ is continuous on $[f, g]$, for all $h_{0} \in[f, g]$ there exists $\delta_{0}\left(h_{0}\right)>\theta$ in $\mathcal{C}[a, b]$ such that whenever $h \in[f, g]$ with $\left|h-h_{0}\right|<\delta_{0}\left(h_{0}\right)$, we have

$$
\left|F(h)-F\left(h_{0}\right)\right|<\epsilon \cdot e .
$$


Define a gauge $\delta$ on $[f, g]$ by $\delta(h)=\frac{\delta_{0}(h)}{2}$, for all $h \in[f, g]$. Let

$$
D=\left\{\left(\left[f, h_{1}\right], t_{1}\right),\left(\left[h_{1}, h_{2}\right], t_{2}\right), \ldots,\left(\left[h_{m-1}, g\right], t_{m}\right)\right\}
$$

and

$$
Q=\left\{\left(\left[f, k_{1}\right], r_{1}\right),\left(\left[k_{1}, k_{2}\right], r_{2}\right), \ldots,\left(\left[k_{q-1}, g\right], r_{q}\right)\right\}
$$

be $\delta$-fine tagged divisions of $[f, g]$. Then there exists a tagged division $D_{0}$ such that $D \ll D_{0}$ and $Q \ll D_{0}$. Now, for every $\left(\left[h_{i-1}, h_{i}\right], t_{i}\right) \in D, f=h_{0}, h_{m}=g, 1 \leq i \leq m$, consider the difference

$$
\Delta\left(h_{i-1}, h_{i}\right)=F\left(t_{i}\right)\left[H\left(h_{i}\right)-H\left(h_{i-1}\right)\right]-S\left(F, H ; P_{i}\right)
$$

where

$$
P_{i}=\left\{\left(\left[z_{j-1}^{(i)}, z_{j}^{(i)}\right], s_{j}^{(i)}\right)\right\}_{j-1}^{n_{i}}, \quad z_{0}^{(i)}=h_{i-1}, z_{n_{i}}^{(i)}=h_{i}
$$

is the refinement of $\left(\left[h_{i-1}, h_{i}\right], t_{i}\right)$ in $D_{0}$. Then

$$
\Delta\left(h_{i-1}, h_{i}\right)=\sum_{j=1}^{n_{i}}\left[F\left(t_{i}\right)-F\left(s_{j}^{(i)}\right)\right]\left[H\left(z_{j}^{(i)}\right)-H\left(z_{j-1}^{(i)}\right)\right] .
$$

Now, $s_{j}^{(i)}, t_{i} \in\left[h_{i-1}, h_{i}\right] \subseteq\left(t_{i}-\delta\left(t_{i}\right), t_{i}+\delta\left(t_{i}\right)\right)$ which implies that

$$
\left|t_{i}-s_{j}^{(i)}\right| \leq\left|h_{i}-h_{i-1}\right|<\delta\left(t_{i}\right)
$$

By continuity of $F$ at $t_{i}$,

$$
\left|s_{j}^{(i)}-t_{i}\right|<\delta\left(t_{i}\right)=\frac{\delta_{0}\left(t_{i}\right)}{2}<\delta_{0}\left(t_{i}\right) \Rightarrow\left|F\left(s_{j}^{(i)}\right)-F\left(t_{i}\right)\right|<\epsilon \cdot e .
$$

So,

$$
\left|\Delta\left(h_{i-1}, h_{i}\right)\right|=\left|\sum_{j=1}^{n_{i}}\left[F\left(t_{i}\right)-F\left(s_{j}^{(i)}\right)\right]\left[H\left(z_{j}^{(i)}\right)-H\left(z_{j-1}^{(i)}\right)\right]\right| .
$$

Hence, by Theorem 10, we have

$$
\begin{aligned}
\mid S(F, H ; D) & -S\left(F, H ; D_{0}\right)|=| \sum_{i=1}^{m} F\left(t_{i}\right)\left[H\left(h_{i}\right)-H\left(h_{i-1}\right)\right]-\sum_{i=1}^{m} S\left(F, H, P_{i}\right) \mid \\
& =\left|\sum_{i=1}^{m}\left\{F\left(t_{i}\right)\left[H\left(h_{i}\right)-H\left(h_{i-1}\right)\right]-S\left(F, H, P_{i}\right)\right\}\right|=\left|\sum_{i=1}^{m} \Delta\left(h_{i-1}, h_{i}\right)\right| \\
& \leq \sum_{i=1}^{m}\left|\Delta\left(h_{i-1}, h_{i}\right)\right|
\end{aligned}
$$


REFERENCES

$$
\begin{aligned}
& =\sum_{i=1}^{m}\left|\sum_{j=1}^{n_{i}}\left[F\left(t_{i}\right)-F\left(s_{j}^{(i)}\right)\right]\left[H\left(z_{j}^{(i)}\right)-H\left(z_{j-1}^{(i)}\right)\right]\right| \\
& \leq \sum_{i=1}^{m}\left(\sum_{j=1}^{n_{i}}\left|F\left(t_{i}\right)-F\left(s_{j}^{(i)}\right)\right|\left|H\left(z_{j}^{(i)}\right)-H\left(z_{j-1}^{(i)}\right)\right|\right) \\
& \leq \sum_{i=1}^{m}\left(\sum_{j=1}^{n_{i}} \frac{\epsilon}{K} \cdot e \cdot\left|H\left(z_{j}^{(i)}\right)-H\left(z_{j-1}^{(i)}\right)\right|\right) \\
& \leq \frac{\epsilon}{K} \cdot e \cdot \sum_{i=1}^{m}\left(\sum_{j=1}^{n_{i}}\left|H\left(z_{j}^{(i)}\right)-H\left(z_{j-1}^{(i)}\right)\right|\right) \\
& \leq \frac{\epsilon}{K} \cdot e \cdot \sum_{i=1}^{m} v\left(H ;\left[h_{i-1}, h_{i}\right]\right) \\
& =\frac{\epsilon}{K} \cdot e \cdot v_{H}<\frac{\epsilon}{K} \cdot e \cdot K<\epsilon \cdot e .
\end{aligned}
$$

By similar argument,

$$
\left|S(F, H ; Q)-S\left(F, H ; D_{0}\right)\right|<\epsilon \cdot e .
$$

Thus,

$$
\begin{aligned}
|S(F, H ; D)-S(F, H ; Q)| & =\left|S(F, H ; D)-S\left(F, H ; D_{0}\right)+S\left(F, H ; D_{0}\right)-S(F, H ; Q)\right| \\
& \leq\left|S(F, H ; D)-S\left(F, H ; D_{0}\right)\right|+\left|S(F, H ; Q)-S\left(F, H ; D_{0}\right)\right| \\
& <\epsilon \cdot e+\epsilon \cdot e \\
& =2 \epsilon \cdot e .
\end{aligned}
$$

By Cauchy criterion, $F \in \mathcal{H} \mathcal{K} \mathcal{S}([f, g], H)$.

\section{Acknowledgements}

This article is funded by CHED-K12 Transition Program.

\section{References}

[1] T. M. Apostol, Mathematical Analysis, 2nd edition, Narosa Publishing House, New Delhi, 2002.

[2] R. G. Bartle and D. R. Sherbert, Introduction to Real Analysis, 4th edition, John Wiley, New York, 2011.

[3] S. S. Cao, The Henstock Integral for Banach-valued Functions, Southeast Asian Bull. Math. 16, N0. 1, (1992), 35-40. 
[4] D. H. Fremlin, Topological Riesz Spaces and Measure Theory, (Cambridge University Press). 978-0-0521-09031-5.

[5] E. Kreyszig, Introductory Functional Analysis with Application, John Wiley and Sons. Inc., New York, 1978.

[6] J. Kurzweil, Generalized Ordinary Differential Equation and Continuous Dependence on a Parameter, CMJ, 7(82) (1957), 418-449.

[7] J. S. Lim, J. H. Yoon, and G. S. Eun, On Henstock-Stieltjes integral, KangweonKyungki Math. J.6, No.1, (1998), 87-96.

[8] S. A. Tikare and M. S. Chaudhary, The Henstock-Stieltjes Integral for Banach Space-valued Functions, Bull. Kerala Math. Assoc. Vol. 6, N0. 2, (2010), 83-92.

[9] F. Ubaidillah, S. Darmawijaya, and Ch. R. Indrati On the Henstock-Kurzweil Integral of $\mathcal{C}[a, b]$ Space-valued Functions, IJMA, 37(9)(2015), 1831-1846. 